\title{
WILEY-VCH
}

DOI: $10.1002 /(($ please add manuscript number $))$

Article type: Communication

\section{D electrophysiological measurements on cells embedded within fiber-reinforced Matrigel}

Natascha Schaefer ${ }^{\S}$, Dieter Janzen ${ }^{\S}$, Ezgi Bakirci, Andrei Hrynevich, Paul D. Dalton, Carmen

\section{Villmann*}

$\S$ These authors have contributed equally to this work

* Corresponding author

Dr. N. Schaefer, Mr. D. Janzen, Prof. C. Villmann

Institute for Clinical Neurobiology,

University Hospital Würzburg,

Versbacherstr. 5, 97078 Würzburg, Germany

E-mail: Villmann_C@ukw.de

Ms. Ezgi Bakirci, Mr. A. Hrynevich, Prof. P.D. Dalton

Department of Functional Materials in Medicine and Dentistry and Bavarian Polymer Institute University Hospital Würzburg

Pleicherwall 2, 97070, Würzburg, Germany

Keywords: melt electrowriting (MEW), 3D electrophysiology, glycine receptor (GlyR)

This is the peer reviewed version of the following article: Schaefer, N., Janzen, D., Bakirci, E., Hrynevich, A., Dalton, P. D., Villmann, C., Adv. Healthcare Mater. 2019, 8, 1801226 which has been published in final form at https://doi.org/10.1002/ adhm.201801226. This article may be used for non-commercial purposes in accordance with Wiley Terms and Conditions for Use of Self-Archived Versions. This article may not be enhanced, enriched or otherwise transformed into a derivative work, without express permission from Wiley or by statutory rights under applicable legislation. Copyright notices must not be removed, obscured or modified. The article must be linked to Wiley's version of record on Wiley Online Library and any embedding, framing or otherwise making available the article or pages thereof by third parties from platforms, services and websites other than Wiley Online Library must be prohibited. 


\section{WILEY-VCH}

\section{Abstract}

2D electrophysiology is often used to determine the electrical properties of neurons, while in the brain, neurons form extensive 3D networks. Thus, performing electrophysiology in a 3D environment provides a closer situation to the physiological condition and serves as a useful tool for various applications in the field of neuroscience. In this study, we established 3D electrophysiology within a fiber-reinforced matrix to enable fast readouts from transfected cells, which are often used as model systems for 2D electrophysiology. Using melt electrowriting of scaffolds to reinforce Matrigel, we performed 3D electrophysiology on a glycine receptortransfected Ltk-11 mouse fibroblast cell line. The glycine receptor is an inhibitory ion channel associated when mutated with impaired neuromotor behavior. The average thickness of the MEW scaffold was $141.4 \pm 5.7 \mu \mathrm{m}$, using $9.7 \pm 0.2 \mu \mathrm{m}$ diameter fibers, and square pore spacings of $100 \mu \mathrm{m}, 200 \mu \mathrm{m}$ and $400 \mu \mathrm{m}$. We demonstrate, for the first time, the electrophysiological characterization of glycine receptor-transfected cells with respect to agonist efficacy and potency in a 3D matrix. With the MEW scaffold reinforcement not interfering with the electrophysiology measurement, this approach can now be further adapted and developed for different kinds of neuronal cultures to study and understand pathological mechanisms under disease conditions. 


\section{WILEY-VCH}

Three-dimensional (3D) matrices and scaffolds are an essential part of numerous areas of biomedical research, including tissue engineering ${ }^{[1]}$, ${\text { cancer } \text { research }^{[2]} \text {, cell expansion }}^{[3]}$ and stem-cell research ${ }^{[4]}$. In addition, the field of biofabrication is introducing an increasing number of ways to create hierarchical structures ${ }^{[5]}$ to better recapitulate the in vivo environment. These include 3D-cell loaded structures with neurons, that are highly sensitive to changes within their environment e.g. growth factor gradients ${ }^{[6]}, \mathrm{ECM}$-derived peptides ${ }^{[7,8]}$, or the stiffness of the matrix ${ }^{[9]}$. Mechanical properties such as the stiffness of the $3 \mathrm{D}$ surrounding environment are known to affect differentiation of certain cells ${ }^{[10]}$, and are believed to be critical for neuronal maturation and neurotransmission ${ }^{[9]}$.

More discrete and higher resolved structures, such as those made with additive manufacturing $(\mathrm{AM})^{[11]}$ and electrojetting technology ${ }^{[12]}$, are promising to provide reproducible conditions. Electrojetting uses electrostatic forces to fabricate monodispersed, nano-micro particles in a simple, versatile and cost-effective method for drug delivery and tissue engineering applications ${ }^{[13]}$. Electrospinning allows processability for polymer solutions and polymer melts ${ }^{[14]}$. In addition, polymer melts can be directly written using a programmable path in a technique known as melt electrowriting $(\mathrm{MEW})^{[15]}$. The precise placement of low-micron diameter fibers that are stackable using additive manufacturing principles are achievable using MEW (Figure 1a, b). The advantages of MEW include solvent-free processing and avoidance of the chaotic fiber deposition often seen in electrospinning. Bioprinting approaches for cell hierarchy have been reinforced with extruded, stiffer structures ${ }^{[16]}$ that reinforce the bioink and aid with in vitro handling ${ }^{[17]}$ and extend a processing window for such hydrogels ${ }^{[16,17]}$. The reinforcing of matrices and bioinks with much smaller, low micron-scale fibers on matrices has recently been of particular interest ${ }^{[18,19]}$. Using $\mathrm{MEW}^{[15]}$, well-ordered, small diameter fibers can be distributed throughout a matrix in low volume fractions and provide significant increase in overall mechanical properties ${ }^{[18-20]}$. Furthermore, the mechanics of MEW fiber-reinforced hydrogels can be modulated further with sinusoidal direct-writing of the fibers ${ }^{[18]}$. Since the 


\section{WILEY-VCH}

fiber diameter made using MEW can be readily changed with the nozzle mass flow rate and/or the collection speed, the diameter of the printed fiber can be significantly altered ${ }^{[21]}$. Therefore, MEW reinforcement of matrices (such as Matrigel shown here) have the potential to regulate the environment of cells through both mechanotransductive ${ }^{[22]}$ or haptotaptic ${ }^{[23]}$ cues. $^{2}$

An experimentally designed in vitro 3D structure for electrophysiological studies requires a relevant receptor model, with the inhibitory glycine receptor (GlyR) used in this study. The GlyR is a pentameric, ligand gated ion channel, which belongs to the superfamily of Cysloop receptors ${ }^{[24]}$. Upon binding of its ligand glycine, the ion channel undergoes conformational changes, which lead to opening of the ion channel pore ${ }^{[25]}$. Changes in chloride conductance can be measured with electrophysiological recordings and provide a reliable readout about functional characteristics of the GlyR, e.g. channel opening/closing, ligand potency, and efficacy ${ }^{[26]}$. In this study, the mouse fibroblast Ltk-11 cell line was used as the underlying cellular model. MEW scaffolds have previously been infiltrated with fibroblasts ${ }^{[27]}$ while the Ltk-11 cells are well-suited for transfection and 2D electrophysiology ${ }^{[28]}$. A fast readout technique that provides the 3D perspective to study cellular proteins under disease conditions as well as an adaption to the more complex neuronal system would be highly useful.

MEW scaffolds with different z-directional pore sizes were investigated and analysed for their mechanical properties with and without Matrigel (Figure 1c). The fiber diameter of the MEW scaffolds was $9.7 \pm 0.2 \mu \mathrm{m}$ while the thickness was $141.4 \pm 5.7 \mu \mathrm{m}$ without Matrigel. The forcep-handling of the dry scaffolds were similar (Figure 1d), but the $400 \mu \mathrm{m}$ pore size became difficult to handle (Figure 1e) when Matrigel was included. The $400 \mu \mathrm{m}$ pore scaffolds rolled up when picked up by forceps and collapsed (Figure 1e, right image), while the $100 \mu \mathrm{m}$ and $200 \mu \mathrm{m}$ pore scaffolds were more stable. An amplitude sweep was performed to determine the linear viscoelastic region of Matrigel (Figure 1f) which was $1 \%$ strain. A lower strain $0.1 \%$ was therefore selected for frequency sweep and confirmed a crosslinked hydrogel (Figure 1g) ${ }^{[29,30]}$. 


\section{WILEY-VCH}

The estimation of living and dead cells of Ltk-11 cells grown in 2D compared to 3D embedded Ltk-11 cells in MEW scaffold reinforced Matrigel revealed no significant differences in the number of live and dead cells (2D live $98.5 \%$, dead $1.5 \%$; 3D live $98.9 \%$, dead $1.1 \% \pm 0.3$; Figure 2a, b). Cell division in 3D starts $48 \mathrm{~h}$ post-seeding, which is around $24 \mathrm{~h}$ later compared to $2 \mathrm{D}$ (data not shown), possibly due to the mechanical properties of the Matrigel. Smaller pores harbor less cells (100 $\mu \mathrm{m}$; Figure 2c, upper images), more cells were present in a $200 \mu \mathrm{m}$ and $400 \mu \mathrm{m}$ scaffolds (Figure 2c, middle and lower panel). Following $24 \mathrm{~h}$ in scaffold, most Ltk11 cells still form clustered structures (Figure 2c). This might be explained by better growth of cells when in contact with neighboring cells ${ }^{[31]}$. Comparing the cell number $24 \mathrm{~h}$ post-seeding with 9 days after seeding, cells colonized and thus crossed each pore due to increase in cell division within the MEW-scaffold/Matrigel complex independent of the pore size used (Figure 2c, right magnified images). Hence, our data are in line with previous results that fibroblasts are able to infiltrate MEW-scaffolds ${ }^{[27]}$.

During cell seeding, transfection and immunostaining procedures, the $200 \mu \mathrm{m}$ scaffold turned out to be the most appropriate scaffold for cell biological assays. Ltk-11 cells can be easily transfected in $2 \mathrm{D}$ cultures. In $3 \mathrm{D}$, neither the calcium phosphate precipitation method based on complex formation of plasmid DNA and calcium phosphate nor lipofectamine transfection based on a hydrophobic complex were successful ${ }^{[32]}$. The calcium phosphate precipitates were unable to enter the Matrigel and remained at the matrix surface. Similarly, the DNAlipofectamine complex did not diffuse into the Matrigel to target the Ltk-11 cells. Therefore, Ltk-11 cells were transfected in $2 \mathrm{D}$ cell cultures and transferred $24 \mathrm{~h}$ post-transfection together with Matrigel into the scaffold (Figure 2d). eGFP served as internal control for transfection efficiency. The GlyR $\alpha 1$ subunit was chosen due to its ability to form homomeric ligand-gated ion channels. Note, every cell which shows green fluorescence successfully expresses eGFP. It is assumed that cells expressing eGFP have also taken up the plasmid DNA for the GlyR. As a proof, immunocytochemical staining was performed to analyze the expression of the GlyR $\alpha 1$ 


\section{WILEY-VCH}

subunit and eGFP in the 3D environment. Colocalization of eGFP and GlyR $\alpha 1$ was observed in the same transfected Ltk-11 cell (Figure 2e). Therefore, electrophysiological experiments were only performed on green fluorescent cells. For electrophysiological purposes, the pore size of $100 \mu \mathrm{m}$ impacted the movements of the pipette tip to reach the cell of interest. Because of this, we continued with the $200 \mu \mathrm{m}$ pore scaffolds for all following experiments. Transfected Ltk-11 cells were used in parallel for studies in 2D or 3D to enable comparison.

Scaffolds were transferred into a recording chamber filled with extracellular buffer (ECS) to enable measurements under physiological conditions (Figure 3a, b). ECS is specific for each cell type. The soft cell/Matrigel/scaffold construct required fixation with an O-ring ${ }^{[33]}$ to facilitate electrophysiological recordings (Figure 3a). The cells were approached with a glass pipette with positive pressure continuously applied to avoid plugging of the patch pipette with Matrigel. Following attachment of the cellular membrane (on-cell configuration), a negative membrane potential of -60 mV was applied. The cellular membrane was disrupted and wholecell measurements were performed applying a concentration series of glycine to determine the glycine concentration $\left(E C_{50}\right)$ where half of the ion channels are open. A pressure-based application system was used to apply the agonist for $250 \mathrm{~ms}$ with a pressure of 1 bar (Octaflow II, ALA Scientific Instruments, Farmingdale, NY, United States) as glycine would otherwise take too long to reach the cell through the viscous Matrigel. After applying glycine, a washing step with ECS for $7250 \mathrm{~ms}$ with a pressure of 1 bar was necessary, otherwise the glycine would stick to the cell surrounding. For comparison with 2D measurements, transfected Ltk-11 cells grown on glass coverslips surrounded by ECS were used. The glycine-induced current at saturating glycine concentration $(1 \mathrm{mM})$ in 2D does not differ in shape to 3D arguing for similar onset and recovery of the ion channels (Figure 3c). However, $I_{\max }$ currents at $1 \mathrm{mM}$ glycine are smaller in 3D than in 2D ( $\left.I_{\max } 2 \mathrm{D}: 3.3 \pm 0.6 \mathrm{nA} ; I_{\max } 3 \mathrm{D}: 2.2 \pm 0.5 \mathrm{nA}\right)$ (Figure 3c-e, Table 1), which can be explained by the viscous Matrigel preventing glycine from reaching GlyRs around the whole cell especially those on the opposite site of the application system. Therefore, not all 


\section{WILEY-VCH}

GlyR channels of the approached cell open at the same time, leading to less chloride ion influx. Measured $E C_{50}$ values showed a slight rightward shift compared to $2 \mathrm{D}\left(E C_{50} 2 \mathrm{D}\right.$ : $110.5 \pm 17.6 \mu \mathrm{M})$ and $3 \mathrm{D}\left(E C_{50} 3 \mathrm{D}: 174.7 \pm 34.3 \mu \mathrm{M}\right)($ Figure $3 \mathrm{e})$. At $300 \mu \mathrm{M}$ of glycine, $I_{300}$ differed significantly (Figure 3d, Table 1). In sum, we have successfully established a 3D electrophysiology approach based on embedded transfected Ltk-11 cells in Matrigel reinforced with MEW scaffolds. This protocol can now be adapted to other cell types. Our data demonstrate small changes in glycine-induced currents as well as for glycine potency. The comparison of $2 \mathrm{D}$ and $3 \mathrm{D}$ cultures of transfected Ltk-11 cells revealed a significant reduction in current amplitudes following application of $300 \mu \mathrm{M}$ glycine but similar current values for all other glycine concentrations applied. Differences in glycine potency determined by the $E C_{50}$ value exhibited a slight rightward shift (magnitude shift is less than twice) of the dose-response curve in $3 \mathrm{D}$ compared to $2 \mathrm{D}$. For whole cell recordings from $2 \mathrm{D}$ cell cultures of transfected HEK293 cells, glycine $E C_{50}$ values between 20-120 $\mu \mathrm{M}$ have been documented from different labs ${ }^{[34]}$. Differences in cell number, number of transiently transfected cells, promoter of DNA plasmid used, and state or passage of the culture have been pointed out to underlie those values. The decrease in glycine potency from $110 \mu \mathrm{M}$ in $2 \mathrm{D}$ towards $175 \mu \mathrm{M}$ in $3 \mathrm{D}$ is however not significant and might result from differences in surrounding matrix density decelerating glycine from fast approaching the cell. Recently, Frega and colleagues also compared 3D to 2D electrophysiology using hippocampal neurons coupled to a microelectrode-array ${ }^{[35]}$. Significant differences with respect to mean burst rates and network burst duration times compared to $2 \mathrm{D}$ electrophysiological measurements were observed ${ }^{[35]}$. Xu et al. could not observe differences in the firing rates and spontaneous inhibitory postsynaptic currents in hippocampal neurons cultured in a $3 \mathrm{D}$ collagen hydrogel but slight changes in the resting membrane potential and the duration of the after-hyperpolarization differed to $2 \mathrm{D}$ cultures ${ }^{[33]}$. Thus, the physiological properties of cells differ between $2 \mathrm{D}$ and $3 \mathrm{D}$ cultures arguing for a requirement of 3D techniques, which are closer to the natural situation than planar cell growth in 2D cell cultures. 


\section{WILEY-VCH}

The electrophysiological measurements of transfected cells embedded within fiber-reinforced Matrigel harbors all advantages of a 3D system such as the mimicking of a physiological tissue environment including the ability to grow in all three dimensions, better access to nutrients and suitable to describe the physiological properties of the cells used. The fiber-reinforcement using MEW scaffolds could in future provide physical cues for cell migration ${ }^{[36]}$ or process guidance $^{[7]}$. Such fiber-reinforced matrices provide a systematic, composite platform that can be adapted to other cell systems comprising multiple circuit information to study the electrophysiological properties of ion channels in a 3D environment.

\section{Experimental section}

\subsection{MEW Process}

MEW scaffolds were fabricated by a previously described custom-built $\mathrm{MEW}^{[37]}$. Medicalgrade PCL (Corbion Inc, Netherlands, PURASORB PC12, Lot\# 1412000249, 03/2015) was used to fabricate the MEW scaffolds. All MEW printing was performed at $21 \pm 2{ }^{\circ} \mathrm{C}$ environment temperature and a humidity of $35 \pm 10 \%$. PCL was heated at $80^{\circ} \mathrm{C}$ in $3 \mathrm{~mL}$ syringe and air pressure was set to 3 bar. A high voltage was applied and after an electrified jet was generated, the G-code that drive the collector was initiated. A large $48 \times 96 \mathrm{~mm}$ rectangular mesh with $100 \mu \mathrm{m}, 200 \mu \mathrm{m}$ or $400 \mu \mathrm{m}$ spacing fiber was direct-written and then cut with an infrared laser to $9 \mathrm{~mm}$ circular disks for use in 24-well plates for in vitro experiments. MEW scaffolds are produced using the following parameters: $25 \mathrm{G}$ nozzle and a $6 \mathrm{kV}$ voltage applied across a $4 \mathrm{~mm}$ collector distance.

\subsection{SEM}

Scanning electron microscopy (Crossbeam 340, Carl Zeiss AG Oberkochen, Germany) was used to image the MEW scaffolds, sputter-coated with a $4 \mathrm{~nm}$ platinum layer. Software from Zeiss (Zeiss SmartSEM Version 6.03) was used for measurement of fiber size.

\subsection{Handling Test}




\section{WILEY-VCH}

MEW scaffolds with and without Matrigel were photographed using forceps to determine the extent of folding. Prior to embedding, the scaffolds were washed with $70 \%$ ethanol, sterile water, and phosphate-buffered saline then dried at room temperature $\left(21 \pm 2{ }^{\circ} \mathrm{C}\right) .120 \mu \mathrm{L}$ MatrigelDMEM mixtures were added on top of scaffolds and left for gelation for $30 \mathrm{~min}$.

\subsection{Mechanical Behavior}

The frequency and amplitude sweep of Matrigel $(n=4)$ was measured using a controlled stress rheometer (Physica MCR301, Anton Paar GmbH, Germany) with a plate-plate configuration (diameter $=25 \mathrm{~mm}) .4 .5 \mathrm{mg} / \mathrm{mL}$ Matrigel $(500 \mathrm{uL})$ samples were added to the center of the lower plate at $4{ }^{\circ} \mathrm{C}$. The upper plate was placed on samples with $0.5 \mathrm{~mm}$ gap. The temperature was increased from $4{ }^{\circ} \mathrm{C}$ to $37^{\circ} \mathrm{C}$ and kept at $37^{\circ} \mathrm{C}$ for $45 \mathrm{~min}$. All the measurements were done at $37^{\circ} \mathrm{C}$. The amplitude sweep was performed between $0.01 \%$ and $100 \%$ strain at $1 \mathrm{~Hz}$. Frequency sweep was performed between $1 \mathrm{~Hz}$ and $50 \mathrm{~Hz}$ at $0.1 \%$ strain.

\subsection{Cell culture}

Ltk-11 mouse fibroblast cells (CRL-10422; ATCC, Manassas, VA, United States) were cultured in Dulbecco's modified eagle medium (DMEM) supplemented with $10 \%$ fetal calf serum, $4 \mathrm{mM}$ GlutaMAX, $1 \mathrm{mM}$ sodium pyruvate, and $50 \mathrm{U} / \mathrm{mL}$ penicillin/streptomycin (Thermo Fisher Scientific, Waltham, United States) under standard growth conditions at $37^{\circ} \mathrm{C}$ and $5 \% \mathrm{CO}_{2}$.

\subsection{Transfection}

Ltk-11 cells were transiently transfected using Lipofectamine 2000 reagent (Thermo Fisher Scientific, Waltham, United States) according to the manufacturer's protocol. In brief, OptiMem (Life Technologies, Darmstadt, Germany) was mixed with either $6 \mu \mathrm{L}$ of lipofectamine or $2 \mu \mathrm{L}(1 \mu \mathrm{g} / \mu \mathrm{L})$ of plasmid DNA and incubated for $5 \mathrm{~min}$ at room temperature before combining. Following a second 15 min incubation period, the mixture was carefully pipetted onto the cells ( $3 \mathrm{~cm}$ dish). Cells were transfected with the GlyR $\alpha 1$ subunit and eGFP (enhanced 


\section{WILEY-VCH}

green fluorescent protein). eGFP was used as an internal transfection efficiency control and fluorescence of eGFP was controlled next day.

\subsection{Scaffold preparation and cell seeding}

Scaffolds were placed into 24 -well plates, washed once with $70 \%$ ethanol, three times with sterile water, and once with sterile PBS. Transfected Ltk-11 cells were detached 1 day after transfection and counted. Cells were either seeded onto 4 coverslips in a $3 \mathrm{~cm}$ dish $(150.000$ cells/dish) or $60-100 \mu \mathrm{L}$ cell suspension (100.000 cells) were mixed with Matrigel (Corning, NY, United States) using a final protein concentration of $4.5-4.8 \mathrm{mg} / \mathrm{mL}$ and pipetted onto scaffolds. After $30 \mathrm{~min}$ incubation at $37^{\circ} \mathrm{C}, 500 \mu \mathrm{L}$ DMEM was added.

\subsection{Immunocytochemical staining}

Glycine receptors were stained to assess cell transfection efficiency. All steps were performed at room temperature. Cells in MEW scaffolds were fixed for 5 min with $2 \%$ paraformaldehyde, washed three times with PBS and blocked/permeabilized for $30 \mathrm{~min}$ with 5\% (v/v) goat serum and $0.2 \%$ Triton X-100 in PBS. Afterwards, cells were incubated for $1 \mathrm{~h}$ with the GlyR $\alpha 1-$ specific primary antibody mAb2b (1:500 in blocking solution; Synaptic Systems, Göttingen, Germany, Cat. No: 146 111). Cells were washed three times with PBS and incubated for 45 min with the secondary Cy3-coupled goat-anti-mouse antibody (1:500 in blocking solution;

Dianova, Hamburg, Germany). Cells were washed three times with PBS, once with $\mathrm{dH}_{2} \mathrm{O}$, and mounted on glass slides with ProLong Glass Antifade Mountant (Thermo Fisher Scientific, Waltham, United States). Mounting Medium contains Hoechst 33342.

\subsection{Cell viability}

The viability of transfected Ltk-11 cells was measured 1 day after seeding in 2D and 3D cultures. Briefly, cells were incubated for 30 min with $2 \mu \mathrm{M}$ Calcein-AM (green/living cells; Thermo Fisher Scientific, Waltham, United States) and $2 \mu \mathrm{M}$ Ethidium homodimer I (red/dead cells; Sigma-Aldrich, St. Louis, Unites States) in PBS. Five images each of three independent samples were used for measuring the live/dead ratio. 


\section{WILEY-VCH}

\subsection{Confocal microscopy and image acquisition}

Fluorescence and cell viability images were acquired with an inverted Olympus IX81 microscope equipped with an Olympus FV1000 confocal laser scanning system, a FVD10 SPD spectral detector, and diode lasers of $405 \mathrm{~nm}$ (Hoechst), $495 \mathrm{~nm}$ (Alexa488), and $550 \mathrm{~nm}$ (Cy3) (Olympus, Tokyo, Japan). All images shown were acquired using an Olympus UPLFLN 40x (oil, numerical aperture: 1.3) or UPLSAPO 10x (air, numerical aperture: 0.4) objective. Phasecontrast images were acquired using a Leica DM IL LED (Leica, Wetzlar, Germany) microscope equipped with a HTC 10 (HTC, New Taipei City, Taiwan). Images were processed with ImageJ (1.52)/Fiji ${ }^{[38]}$.

\subsection{Electrophysiology}

The patch clamp technique was used to measure current amplitudes $(I)$ of transfected Ltk-11 cells in 2D (coverslips) and 3D (Matrigel reinforced with MEW scaffolds) conditions $48 \mathrm{~h}$ after transfection in a whole-cell configuration mode. Currents were amplified using an EPC10 amplifier and the software Patchmaster NEXT (HEKA, Lambrecht, Germany). For doseresponse curves to determine the glycine $E C_{50}$, seven glycine concentrations were used (2D: 3 , $10,30,60,100,300,1000 \mu \mathrm{M}$; and 3D: 10, 30, 60, 100, 300, $6001000 \mu \mathrm{M})$ were applied for $250 \mathrm{~ms}$ with a pressure of $1 \mathrm{bar}$, followed by application of ECS for $7250 \mathrm{~ms}$ using an Octaflow II system (ALA Scientific Instruments, Farmingdale, NY, United States). The extracellular buffer consisted of (in mM): $137 \mathrm{NaCl}, 5.4 \mathrm{KCl}, 1.8 \mathrm{CaCl}_{2}, 1 \mathrm{MgCl}_{2}, 5 \mathrm{HEPES}$, pH adjusted to 7.4 with $\mathrm{NaOH}$. The intracellular buffer contained (in $\mathrm{mM}$ ): $120 \mathrm{CsCl}, 20 \mathrm{~N}(\mathrm{Et}){ }_{4} \mathrm{Cl}, 1 \mathrm{CaCl}_{2}$, $2 \mathrm{MgCl}_{2}, 11$ EGTA, 10 HEPES, pH adjusted to 7.2 with $\mathrm{CsOH}$. Cells were held at $-60 \mathrm{mV}$. Recording pipettes were manufactured from borosilicate capillaries and were pulled with the help of a P97 horizontal puller (Sutter Instrument, Novato, CA, United States). Recording pipettes had an open resistance of 4-6 M . All measurements were performed at $21^{\circ} \mathrm{C}$.

\subsection{Statistical analysis}




\section{WILEY-VCH}

Electrophysiological data was analyzed using OriginPro 2018b (OriginLab, Northampton, MA, United States). A non-linear algorithm was used to construct dose-response curves from peak current amplitudes obtained with seven appropriately spaced concentrations in the range of 10$1000 \mu \mathrm{M}$ glycine. The following Hill equation was used: $I=I_{\max }{ }^{*} c^{n H} /\left(c^{n H}+E C_{50}{ }^{n H}\right) . I$ refers to the current amplitude at the given agonist concentration $c, I_{\max }$ is the current amplitude at a saturating agonist concentration, $E C_{50}$ refers to the agonist concentration evoking half-maximal current responses and $n_{H}$ is the Hill coefficient.

Statistical analysis was performed using calculation of mean values, followed by determination of the standard deviation (SD) or standard error of the mean (SEM) (electrophysiological measurements - calculation from 8-10 measured cells out of three or more independent transfections were performed; amplitude and frequency sweep-calculated from four independent replicates). Subsequent student's t-test was used to evaluate the probability values $=p$ values: $p$-values were set as follows: ${ }^{*} p \leq 0.05 ; * * p \leq 0.01$ and ${ }^{* * *} p \leq 0.001$

\section{Supporting Information}

Supporting Information is available online from the Wiley Online Library or from the author.

\section{Acknowledgements}

The authors state no conflict of interest. This work was supported by the German Research Foundation (DFG) as part of the collaborative research centre SFB TR225 (sub-project B01). INST 93/940-1 and the State Major Instrumentation Program, which financed the Scanning electron microscope used in this study (INST 105022/58-1 FUGG), is gratefully acknowledged. The MEW 3D printer used for this study was built by AH. The authors appreciate the assistance in initial scaffold manufacture by Almoatazbellah Youssef. 


\section{WILEY-VCH}

Received: ((will be filled in by the editorial staff))

Revised: ((will be filled in by the editorial staff)) Published online: ((will be filled in by the editorial staff))

\section{References}

[1] N. Annabi, K. Tsang, S. M. Mithieux, M. Nikkhah, A. Ameri, A. Khademhosseini, A. S. Weiss, Adv Funct Mater 2013, 23.

[2] D. W. Hutmacher, R. E. Horch, D. Loessner, S. Rizzi, S. Sieh, J. C. Reichert, J. A. Clements, J. P. Beier, A. Arkudas, O. Bleiziffer, U. Kneser, Journal of Cellular and Molecular Medicine 2009, 13, 1417.

[3] B. Delalat, F. Harding, B. Gundsambuu, E. M. De-Juan-Pardo, F. M. Wunner, M. L. Wille, M. Jasieniak, K. A. L. Malatesta, H. J. Griesser, A. Simula, D. W. Hutmacher, N. H. Voelcker, S. C. Barry, Biomaterials 2017, 140, 58.

[4] R. A. Marklein, J. A. Burdick, Adv Mater 2010, 22, 175.

[5] J. Groll, T. Boland, T. Blunk, J. A. Burdick, D. W. Cho, P. D. Dalton, B. Derby, G. Forgacs, Q. Li, V. A. Mironov, L. Moroni, M. Nakamura, W. Shu, S. Takeuchi, G. Vozzi, T. B. Woodfield, T. Xu, J. J. Yoo, J. Malda, Biofabrication 2016, 8, 013001.

[6] X. Cao, M. S. Shoichet, Neuroscience 2001, 103, 831.

[7] P. D. Dalton, J. Mey, Front Biosci (Landmark Ed) 2009, 14, 769.

[8] Y. Luo, M. S. Shoichet, Biomacromolecules 2004, 5, 2315.

[9] G. Palazzolo, N. Broguiere, O. Cenciarelli, H. Dermutz, M. Zenobi-Wong, Tissue Eng Part A 2015, 21, 2177.

[10] S. H. Oh, D. B. An, T. H. Kim, J. H. Lee, Acta Biomater 2016, 35, 23.

[11] A. Youssef, S. J. Hollister, P. D. Dalton, Biofabrication 2017, 9, 012002.

[12] P. Fattahi, A. Borhan, M.-R. Abidian, presented at 2013 6th International IEEE/EMBS Conference on Neural Engineering (NER) 2013, 6-8 Nov. 2013, 953.

[13] P. Fattahi, A. Borhan, M. R. Abidian, Adv Mater 2013, 25, 4555.

[14] T. J. Sill, H. A. von Recum, Biomaterials 2008, 29, 1989.

[15] P. D. Dalton, Current Opinion in Biomedical Engineering 2017, $2,49$.

[16] J. Malda, J. Visser, F. P. Melchels, T. Jungst, W. E. Hennink, W. J. Dhert, J. Groll, D. W. Hutmacher, Adv Mater 2013, 25, 5011.

[17] N. V. Mekhileri, K. S. Lim, G. C. J. Brown, I. Mutreja, B. S. Schon, G. J. Hooper, T. B. F. Woodfield, Biofabrication 2018, 10, 024103.

[18] O. Bas, D. D'Angella, J. G. Baldwin, N. J. Castro, F. M. Wunner, N. T. Saidy, S. Kollmannsberger, A. Reali, E. Rank, E. M. De-Juan-Pardo, D. W. Hutmacher, ACS Appl Mater Interfaces 2017, 9, 29430.

[19] J. Visser, F. P. Melchels, J. E. Jeon, E. M. van Bussel, L. S. Kimpton, H. M. Byrne, W. J. Dhert, P. D. Dalton, D. W. Hutmacher, J. Malda, Nature Comm 2015, 6, 6933.

[20] a) O. Bas, E. M. De-Juan-Pardo, M. P. Chhaya, F. M. Wunner, J. E. Jeon, T. J. Klein, D. W. Hutmacher, European Polymer Journal 2015, 72, 451; b) M. de Ruijter, A. Hrynevich, J. N. Haigh, G. Hochleitner, M. Castilho, J. Groll, J. Malda, P. D. Dalton, Small 2018, 14.

[21] A. Hrynevich, B. S. Elci, J. N. Haigh, R. McMaster, A. Youssef, C. Blum, T. Blunk, G. Hochleitner, J. Groll, P. D. Dalton, Small 2018, 14, e1800232.

[22] B. M. Baker, C. S. Chen, J Cell Sci 2012, 125, 3015.

[23] R. M. Smeal, R. Rabbitt, R. Biran, P. A. Tresco, Ann Biomed Eng 2005, 33, 376.

[24] J. W. Lynch, Physiol Rev 2004, 84, 1051.

[25] J. Du, W. Lu, S. Wu, Y. Cheng, E. Gouaux, Nature 2015, 526, 224. 


\section{WILEY-VCH}

[26] a) N. Schaefer, A. Berger, J. van Brederode, F. Zheng, Y. Zhang, S. Leacock, L. Littau, S. Jablonka, S. Malhotra, M. Topf, F. Winter, D. Davydova, J. W. Lynch, C. J. Paige, C. Alzheimer, R. J. Harvey, C. Villmann, J Neurosci 2017, 37, 7948; b) N. Schaefer, C. J. Kluck, K. L. Price, H. Meiselbach, N. Vornberger, S. Schwarzinger, S. Hartmann, G. Langlhofer, S. Schulz, N. Schlegel, K. Brockmann, B. Lynch, C. M. Becker, S. C. Lummis, C. Villmann, J Neurosci 2015, 35, 422.

[27] B. L. Farrugia, T. D. Brown, Z. Upton, D. W. Hutmacher, P. D. Dalton, T. R. Dargaville, Biofabrication 2013, 5, 025001.

[28] C. F. Valenzuela, R. A. Cardoso, M. J. Wick, J. L. Weiner, T. V. Dunwiddie, R. A. Harris, Alcoholism, Clinical and Experimental Research 1998, 22, 1132.

[29] A. S. Hoffman, Adv Drug Deliv Rev 2002, 54, 3.

[30] Y. Hong, T. V. Chirila, S. Vijayasekaran, P. D. Dalton, S. G. Tahija, M. J. Cuypers, I. J. Constable, J Biomed Mater Res 1996, 30, 441.

[31] M. Natoli, B. D. Leoni, I. D'Agnano, M. D'Onofrio, R. Brandi, I. Arisi, F. Zucco, A. Felsani, J Cell Physiol 2011, 226, 1531.

[32] a) C. Chen, H. Okayama, Mol Cell Biol 1987, 7, 2745; b) P. L. Felgner, T. R. Gadek, M. Holm, R. Roman, H. W. Chan, M. Wenz, J. P. Northrop, G. M. Ringold, M. Danielsen, Proc Natl Acad Sci U S A 1987, 84, 7413; c) P. L. Felgner, G. M. Ringold, Nature 1989, 337, 387.

[33] T. Xu, P. Molnar, C. Gregory, M. Das, T. Boland, J. J. Hickman, Biomaterials 2009, 30, 4377.

[34] S. Fucile, D. de Saint Jan, B. David-Watine, H. Korn, P. Bregestovski, J Physiol 1999, 517 ( Pt 2), 369.

[35] M. Frega, M. Tedesco, P. Massobrio, M. Pesce, S. Martinoia, Scientific Reports 2014, 4, 5489.

[36] H. G. Sundararaghavan, J. A. Burdick, Biomacromolecules 2011, 12, 2344.

[37] T. D. Brown, P. D. Dalton, D. W. Hutmacher, Adv Mater 2011, 23, 5651.

[38] a) J. Schindelin, I. Arganda-Carreras, E. Frise, V. Kaynig, M. Longair, T. Pietzsch, S. Preibisch, C. Rueden, S. Saalfeld, B. Schmid, J. Y. Tinevez, D. J. White, V. Hartenstein, K. Eliceiri, P. Tomancak, A. Cardona, Nat Methods 2012, 9, 676; b) J. Schindelin, C. T. Rueden, M. C. Hiner, K. W. Eliceiri, Molecular Reproduction and Development 2015, 82, 518; c) C. A. Schneider, W. S. Rasband, K. W. Eliceiri, Nat Methods 2012, 9, 671. 


\section{WILEY-VCH}

\section{Figures}
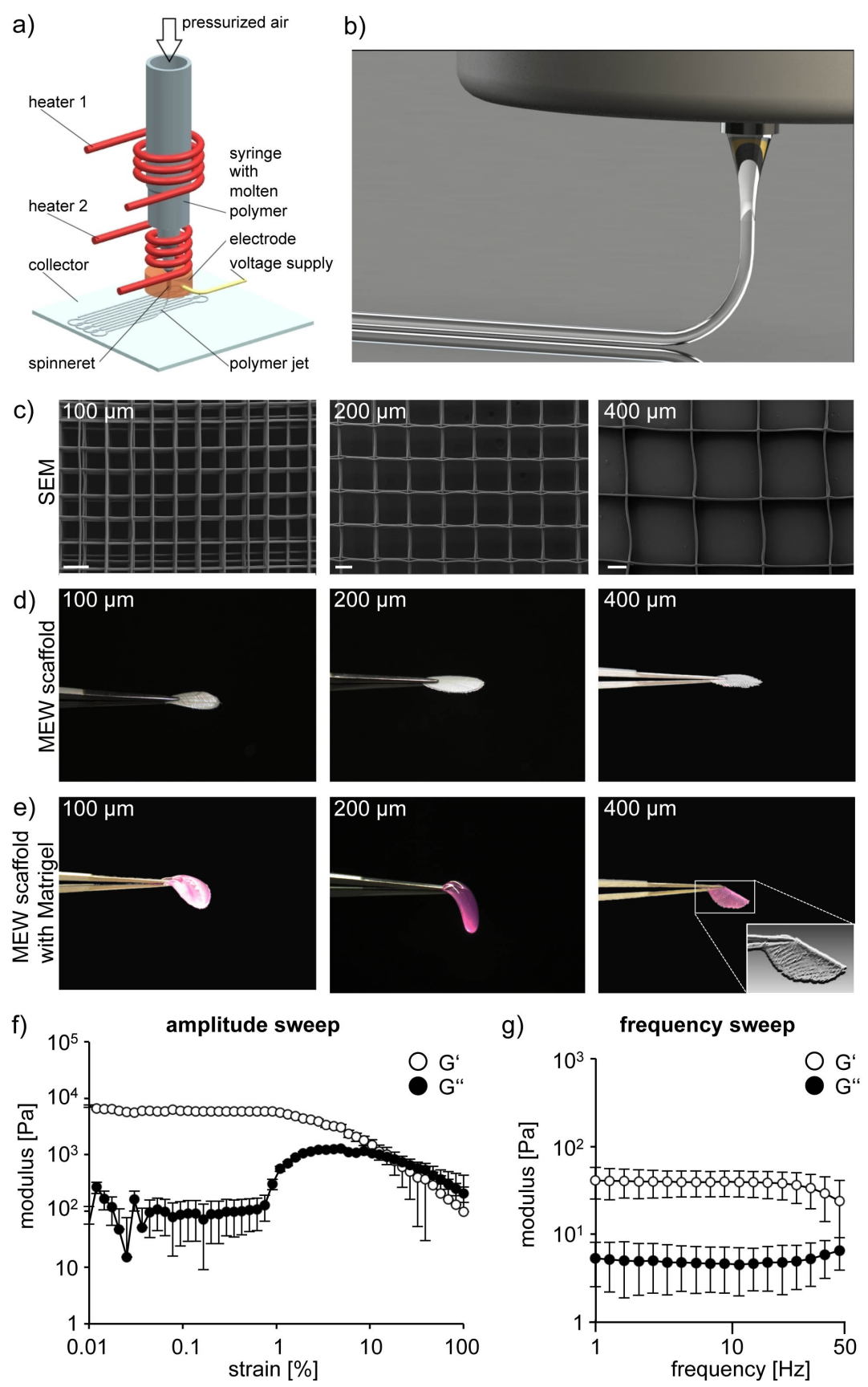

Figure 1. Handling of different MEW-scaffolds with and without matrix. a) Schematic of the MEW head depicting how the polymer melt is heated and the nozzle directed over the surface. b) Rendering of the electrified molten jet as it comes out of the nozzle and forms a fiber that is direct-written layer-by-layer for the reinforcing MEW-scaffold. c) Scanning electron microscopy images of MEW-scaffold with different pores of $100 \mu \mathrm{m}, 200 \mu \mathrm{m}$ and $400 \mu \mathrm{m}$, diameter of scaffolds is $9.8 \mathrm{~mm}$. d) Same scaffolds as in c) held with forceps to investigate stability during handling. e) Scaffolds as in c) and d) reinforced with Matrigel. Note, MEWscaffolds allow handling of ultra-weak matrices, but handling becomes difficult if the pore size increase, e.g. $400 \mu \mathrm{m}$. The collapse of the $400 \mu \mathrm{m}$ scaffold is enlarged in lower right image; basrelief filter used. f) Amplitude and g) Frequency sweep, from $n=4$ independent experiments, error bars represent standard deviation (SD). 


\section{WILEY-VCH}

a)
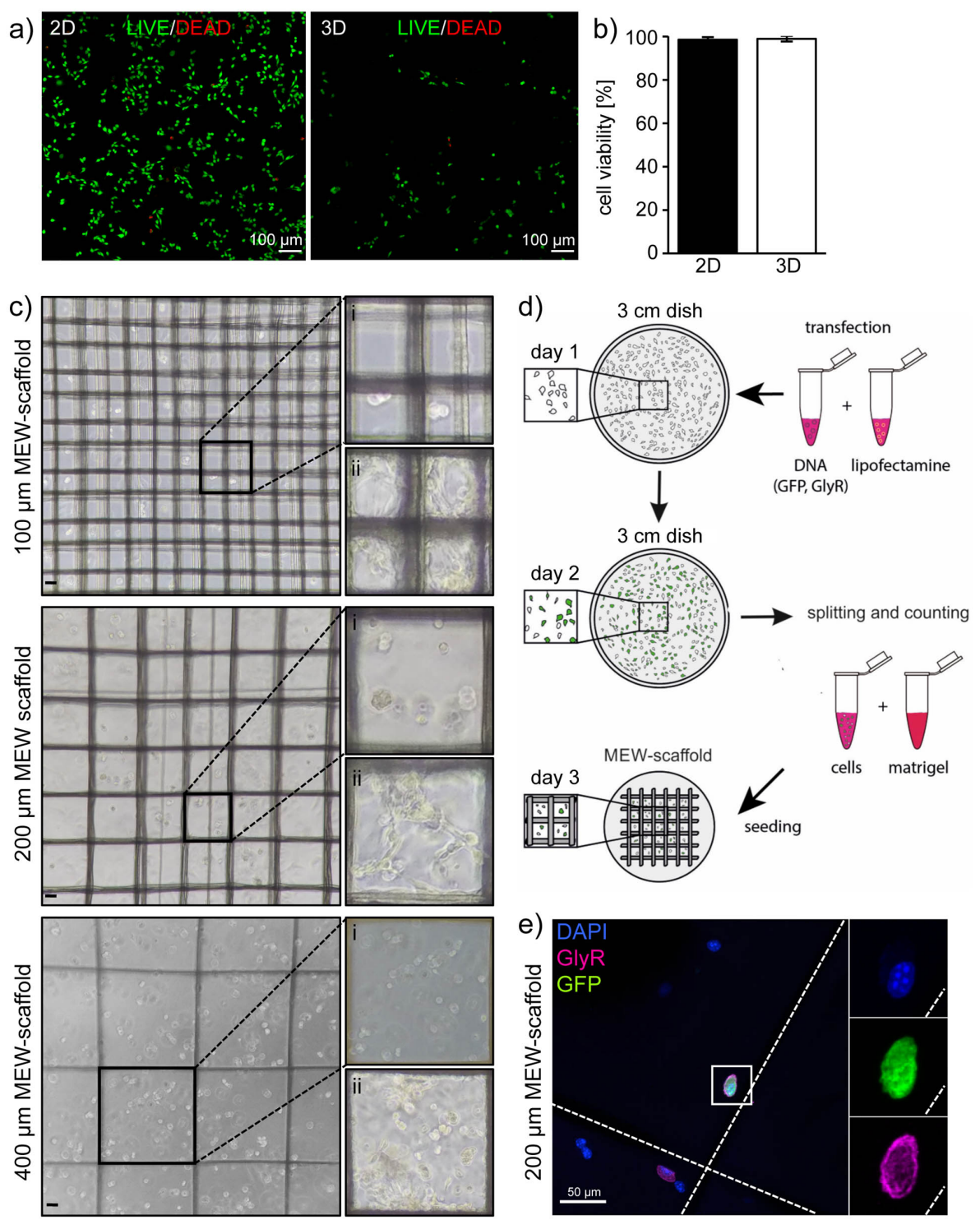

Figure 2. Ltk-11 cells embedded in Matrigel grow in different MEW scaffold sizes and express protein of interest. a) Live/dead assay in 2D cultures and 3D cultures of Ltk-11 cells, live green, dead - red, scale bar refers to $100 \mu \mathrm{m}$; three independent experiments were performed for $2 \mathrm{D}$ and $3 \mathrm{D}$ cultures, 15 images with 5 images per experiment were counted with a total number of counted cells in $2 \mathrm{D}=10115,3 \mathrm{D}=1955)$, b) Quantification of cell viability in 2D and 3D, c) $100 \mu \mathrm{m}, 200 \mu \mathrm{m}$, and $400 \mu \mathrm{m}$ scaffolds side chain length at day 1 after seeding with Ltk-11 cells. A representative pore is shown in magnification at the right with (i) $24 \mathrm{~h}$ after seeding, lower magnified image day 9 after seeding (ii). Scale bar in each picture resembles $50 \mu \mathrm{m}$. d) Exemplary working schedule: day 1 - Ltk-11 cell seeding and transfection with eGFP and GlyR using lipofectamine; day 2 - proof for eGFP positive cells, mixing with Matrigel, and seeding into MEW scaffold; day 3 - scaffold with transfected Ltk-11 cells. e) Immunocytochemical staining of transfected Ltk-11 cells $24 \mathrm{~h}$ after seeding in 3D, $200 \mu \mathrm{m}$ scaffold expressing eGFP (green) and GlyR $\alpha 1$ (magenta). Cell nuclei were stained with Hoechst. Scale bar in each picture resembles $50 \mu \mathrm{m}$. All experiments have been performed at least three times. Representative images are shown. 


\section{WILEY-VCH}

a)

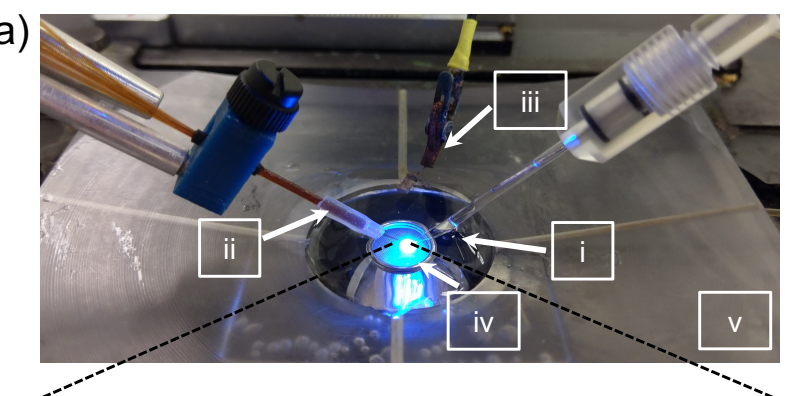

i patch pipette

ii agonist application

iii ground electrode

iv scaffold with

load (O-ring)

$\mathbf{v}$ recording chamber

filled with ECS

b)

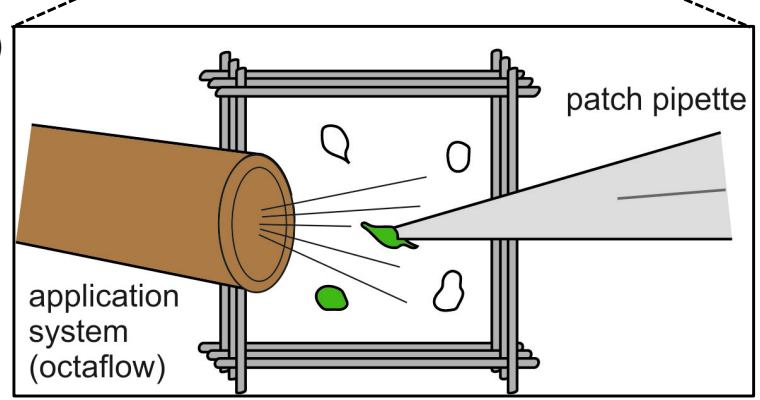

c)

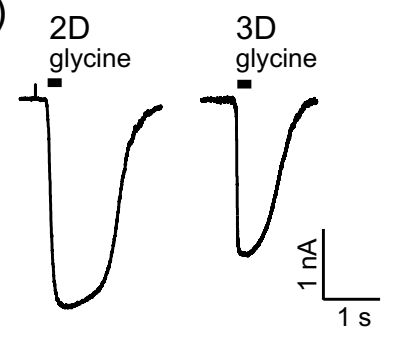

d) $57 \quad 2 \mathrm{D}$
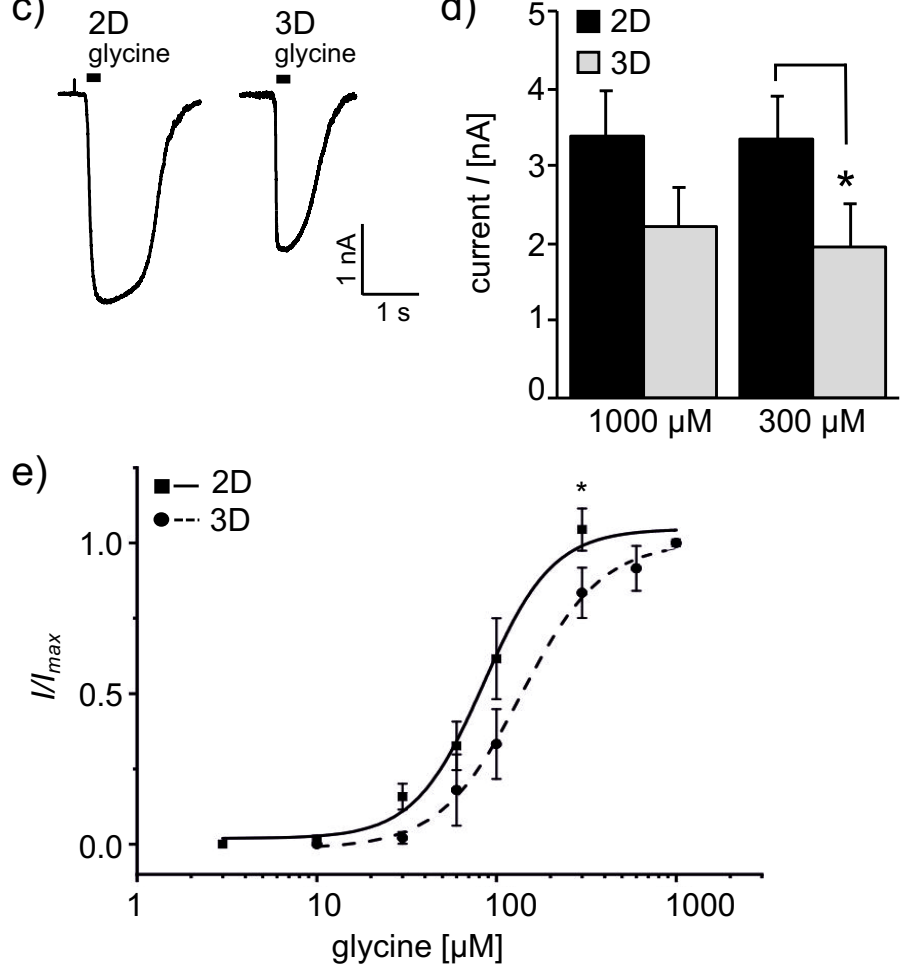

Figure 3. 3D electrophysiological recordings from Ltk-11 cells grown in Matrigel reinforced with MEW-scaffolds. a) Image of the electrophysiological recording chamber with labeled iv. b) Cartoon of the recording from transfected Ltk-11 cells (cotransfected with eGFP, green) grown in MEW scaffolds. c) Representative current traces from 2D and 3D recordings. d) Bar diagram representing $I$ values at $300 \mu \mathrm{M}$ glycine \pm SEM (2D black with $n=10$, 3D grey with $n=8 ; * \mathrm{p} \leq 0.05$ ). e) Dose-response curves obtained from 2D (black squares and full line; $n=10$ ) and 3D (black circles and dotted line; $n=8$ ) measurements to determine the $E C_{50}$. Cells used for data analysis $(\mathrm{n}=8-10)$ have been recorded within three independent experiments. 
Tables

Table 1. Electrophysiological properties of GlyRs determined from reinforced MEW-scaffolds

\begin{tabular}{ccccc}
\hline & $E C_{50}[\mu \mathrm{M}]$ & $n_{H}$ & $I_{\max }[\mathrm{nA}]$ & $n$ \\
\hline 2D & $110.5 \pm 17.6$ & $3.9 \pm 0.5$ & $3.3 \pm 0.6$ & 10 \\
3D & $174.7 \pm 34.3$ & $4.1 \pm 0.7$ & $2.2 \pm 0.5$ & 8 \\
\hline \multicolumn{7}{l}{ Values are presented \pm standard error of the mean (SEM). }
\end{tabular}




\section{WILEY-VCH}

The table of contents entry should be 50-60 words long, and the first phrase should be bold.

Melt electrowriting (MEW) of scaffolds was used to reinforce Matrigel ${ }^{\mathrm{TM}}$ and allow 3D electrophysiology on transfected fibroblast cells. A square pore spacing of $200 \mu \mathrm{m}$ allowed proper cells growth, handling during cell staining, and good cell accessibility by the recording electrode. This approach provides a fundamental milestone to further develop electrophysiology in 3D neuronal networks.

Keyword

3D electrophysiology

Natascha Schaefer§${ }^{\S}$ Dieter Janzen`, Ezgi Bakirci, Andrei Hrynevich, Paul D. Dalton, Carmen Villmann*

Title

3D electrophysiological measurements on cells embedded within fiber-reinforced Matrigel

ToC figure ((Please choose one size: $55 \mathrm{~mm}$ broad $\times 50 \mathrm{~mm}$ high or $110 \mathrm{~mm}$ broad $\times 20 \mathrm{~mm}$ high. Please do not use any other dimensions))

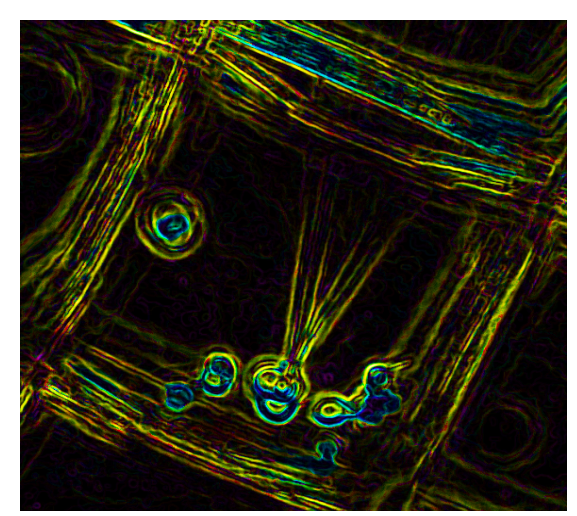

Image shows the recording pipette attached to a patched Ltk-11 cell. Cells are embedded in Matrigel reinforced by MEW scaffolds.

Copyright WILEY-VCH Verlag GmbH \& Co. KGaA, 69469 Weinheim, Germany, 2016. 